\title{
Kosmopolitische Kommunikationswissenschaft: Plädoyer für eine ,tiefe Internationalisierung“ des Fachs in Deutschland
}

\author{
Ein wissenschaftspolitisches Positionspapier
}

\author{
Hanan Badr • Markus Behmer · Susanne Fengler • Anke Fiedler • \\ Anne Grüne - Kai Hafez - Oliver Hahn · Kefa Hamidi • \\ Thomas Hanitzsch • Christine Horz • Beate Illg • Anna Litvinenko • \\ Martin Löffelholz • Melanie Radue · Carola Richter · Barbara Thomaß • \\ Florian Töpfl \\ Online publiziert: 29. Juni 2020 \\ (C) Der/die Autor(en) 2020, korrigierte Publikation 2020
}

H. Badr · A. Fiedler · A. Litvinenko · Prof. Dr. C. Richter $(\bowtie)$

Institut für Publizistik- und Kommunikationswissenschaft, Freie Universität Berlin,

Garystr. 55, 14195 Berlin, Deutschland

E-Mail: carola.richter@fu-berlin.de

\section{Behmer}

Universität Bamberg, Bamberg, Deutschland

S. Fengler

TU Dortmund, Dortmund, Deutschland

A. Grüne · K. Hafez

Universität Erfurt, Erfurt, Deutschland

O. Hahn · F. Töpfl

Universität Passau, Passau, Deutschland

K. Hamidi

Universität Leipzig, Leipzig, Deutschland

T. Hanitzsch

Universität München, München, Deutschland

C. Horz

TH Köln, Köln, Deutschland

B. Illg

Jade-Hochschule Wilhelmshaven, Wilhelmshaven, Deutschland

M. Löffelholz

TU Ilmenau, Ilmenau, Deutschland

M. Radue

Universität Erlangen-Nürnberg, Nürnberg, Deutschland

B. Thomaß

Ruhr-Universität Bochum, Bochum, Deutschland 


\section{Einleitung}

Internationalisierung ist zwar ein verbreitetes Schlagwort im heutigen akademischen Betrieb, und keiner bestreitet dessen Wichtigkeit, doch wie internationalisiert ist die deutsche Kommunikationswissenschaft wirklich? Manche deutschen Hochschulen verstehen unter Internationalisierung lediglich ein (kommunikationswissenschaftliches) englischsprachiges Lehrangebot, andere das Publizieren in englischsprachigen Fachzeitschriften. Dass dies zu kurz greift, versteht sich von selbst. Aber welche internationale Reichweite hat die deutsche Scientific community bisher tatsächlich erzielt? Welchen Stellenwert hat die international vergleichende Forschung, haben inter- und transkulturelle Perspektiven im Fach? Wie steht es um die internationale Anschlussfähigkeit der deutschen Kommunikationswissenschaft? International komparative und kollaborative Forschung und die Einnahme inter-/transkultureller Perspektiven sind Querschnittsaufträge für alle Forschungsfelder des Fachs und können nicht nur beschränkt sein auf so explizit getaufte Netzwerke und Fachgruppen der internationalen und transkulturellen Kommunikation unter dem Dach wissenschaftlicher Fachgesellschaften. Dieses Positionspapier plädiert daher für eine ,tiefe Internationalisierung“ der deutschen Kommunikationswissenschaft.

\section{Status quo, Defizite und Desiderata}

In den vergangenen zwei Jahrzehnten hatte die deutsche Kommunikationswissenschaft erhebliche Fortschritte in Sachen Internationalisierung zu verzeichnen. So gab es an der Wende zum 21. Jahrhundert in Deutschland noch so gut wie keine Lehrstühle oder Professuren mit einer internationalen/inter-/transkulturellen und/ oder international komparativen Denomination. Initiativen wie die Herausgabe des Internationalen Handbuchs Medien am Hans-Bredow-Institut ab 1957, die Gründung des Erich-Brost-Instituts 1991 in Dortmund oder des European Journalism Fellowship-Programms 1999 in Berlin waren bis dato die wenigen Meilensteine auf dem Weg, den Blick über nationale Grenzen hinaus zu weiten. Heute sind entsprechende akademische Strukturen zumindest an einigen deutschen Hochschulen vorhanden - und zwar nicht nur in Metropolen, sondern auch an kleineren Universitäten und Hochschulen. Darüber hinaus beschäftigen sich auch einige Lehrstühle und Professuren ohne explizite internationale Denomination schwerpunktmäßig mit internationalen Fragestellungen. Nicht zuletzt die aus einem Netzwerk (2008) hervorgegangene, 2010 gegründete Fachgruppe „Internationale und Interkulturelle Kommunikation“ der Deutschen Gesellschaft für Publizistik- und Kommunikationswissenschaft (DGPuK) repräsentiert einen gewissen Aufbruch in einer Scientific community, die bis dahin zwar bekannt für ihre theoretischen und methodologischen Beiträge zur Kommunikationswissenschaft war, nicht aber für eine nachhaltige Beschäftigung mit internationalen Forschungsfragen oder eine aktive und systematische Einbindung internationaler Forscher*innen. 
Seit dem ersten Internationalisierungspapier der DGPuK von $2004^{1}$ sind zudem erhebliche Fortschritte bei der Platzierung der deutschen Kommunikationswissenschaft im globalen Wissenschaftskontext zu verzeichnen. Deutsche Wissenschaftler*innen publizieren in renommierten internationalen Fachverlagen, und sie sind in internationalen Fachgesellschaften sowie in Fachzeitschriften wie auch in deren Herausgeber*innengremien prominent vertreten. Selbst Fachzeitschriften mit einem explizit internationalen und transkulturellen Schwerpunkt, wie das Global Media Journal - German Edition, wurden gegründet. Ferner haben sich Master-Studiengänge (in englischer, französischer und deutscher Sprache) etabliert, die dezidiert auf eine internationale Studierendenklientel abzielen. Zudem zählen die deutschen Delegationen mittlerweile zahlenmäßig zu den größten auf den Jahrestagungen der internationalen Fachgesellschaften wie der ICA und der IAMCR.

Dieser vielversprechende strukturelle Aufbruch geht aber noch immer zu selten mit einer inhaltlichen Beschäftigung mit „tiefen“ internationalen Fragestellungen einher. Auch nach Jahrzehnten der Globalisierung des Wissenschaftssystems zeigt nämlich selbst ein kurzer Blick auf den Forschungsstand, dass bei Weitem die meisten Studien, die wir üblicherweise als „,bahnbrechend“ oder „,wegbereitend“ betrachten, von Autor*innen aus englischsprachigen Ländern verfasst wurden (und im deutschen Kontext rechnen wir noch einige deutschsprachige Autor*innen dazu). Diese Schieflage in der Zuweisung von Relevanz in der Forschung hat auch Konsequenzen für unser Verständnis von Medien und Kommunikationsprozessen. Die bisher in der deutschen Kommunikationswissenschaft praktizierte internationale Forschung problematisiert die Gegenstände der Betrachtung tendenziell noch immer aus einer anglo-amerikanischen und eurozentrischen Perspektive. Damit geraten die Realitäten der westlichen Länder zum Prisma, durch das die Forschung „Normalität“ hinsichtlich unseres globalen Verständnisses von Medien und Kommunikationsprozessen konstruiert.

Dies bringt mit sich, dass auch die Internationalisierung der Lehrinhalte häufig einseitig ist: Selbst dort, wo die inhaltliche Internationalisierung ernst genommen wird, ist in den meisten Fällen eine Konzentration auf westliche Länder bzw. ein Industriestaaten-Bias zu erkennen. Dieser Bias wird auch im Bereich der Коттиnikation für sozialen Wandel kritisiert, der sich auf die UN-Agenda 2030 für nachhaltige Entwicklung bezieht und gesellschaftliche Transformationsprozesse in ihrer globalen Interdependenz untersucht. Die Agenda drückt zwar die Ansicht aus, dass sich globale Herausforderungen nur gemeinsam mit allen Nationen lösen lassen, dennoch ist ein großer Teil des wissenschaftlichen Diskurses und das Verständnis davon, was (positive) Entwicklung bedeutet, immer noch stark von eurozentrischen Perspektiven geprägt. Euro-amerikanische Vergleiche sind wichtig, sie blenden aber einen Großteil der Welt aus. Ähnliches gilt für Forschung und Lehre zu transkulturellen Kommunikationsphänomenen, die sich zudem eng an innenpolitische Interessen anlehnt und sich dadurch selbst verengt.

\footnotetext{
1 Abrufbar unter: https://www.researchgate.net/profile/Teresa_Naab/publication/292991053_How_to_ go_international_DGPuK-Wegweiser_Publizieren_und_Referieren_in_der_internationalen_ Kommunikationswissenschaft/links/57fcb2ad08ae329c3d4afcab/How-to-go-international-DGPuKWegweiser-Publizieren-und-Referieren-in-der-internationalen-Kommunikationswissenschaft.pdf.
} 
Auch wenn gerade die Autor*innen dieses Beitrags etliche Projekte auf den Weg gebracht haben, die diese inhaltliche Verengung aufbrechen wollen: ${ }^{2}$ Die Wissenschaftler*innen, die sich mit Kommunikation jenseits der westlichen Welt und Mediensystemen oder Kommunikationsprozessen in Ländern Asiens, Afrikas oder Lateinamerikas befassen, wo etwa drei Viertel der Weltbevölkerung leben, bewegen sich noch immer in einer Nische. Viele dieser Themen erfreuen sich zwar einer rasanten Nachfrage bei Studierenden und auch beim wissenschaftlichen Nachwuchs. Dessen akademische Berufsperspektiven sind aber stark eingeschränkt und bestehen aus Zeitverträgen und begrenzten Drittmittelprojekten statt aus entsprechend ausgerichteten (Junior-)Professuren. Zwischen der Relevanz internationaler Themen und dem oft prekären Arbeitsalltag derjenigen, die sie in Deutschland erforschen wollen, klafft eine eklatante soziale Lücke. Auch der Anteil von Wissenschaftler*innen aus dem Globalen Süden, die diese Perspektiven stärken könnten, ist in der deutschen Kommunikationswissenschaft bisher marginal.

Ohne eine konzeptionelle inhaltliche Neuausrichtung im Kontext einer ,tiefen Internationalisierung“ läuft die deutsche Kommunikationswissenschaft Gefahr, die Anschlussfähigkeit an eine global relevante Kommunikationsforschung zu verspielen. Nach den ersten Schritten einer internationalen Öffnung unserer Wissenschaft in den vergangenen Jahrzehnten ist es nun erforderlich, einen Prozess voranzutreiben, der sich durch mehr Forschung und Lehre über internationale Medien und Kommunikationsprozesse auszeichnet und der durch wissenschaftspolitische Unterstützung und nachhaltige Investitionen abgestützt werden muss.

\section{3 ,Ent-Westernisierung“ und Dezentrierung statt nur ,globale Injektionen“"}

Die Welt entwickelt sich rasant polyzentrisch, der westliche Anteil an der Weltbevölkerung wird immer kleiner, und die westliche Dominanz in den Globalisierungsprozessen schwindet. Auch die deutsche Kommunikationswissenschaft muss sich diesen globalen Herausforderungen stellen. Die ,globalen Injektionen“, die es aktuell bereits gibt, reichen da nicht aus. Leider steht in der deutschen Akademia anstelle eines gesteigerten Weltinteresses noch immer der Wunsch nach einem „Export“ der eigenen Forschungstraditionen im Vordergrund. Ein normativ gerichteter Wissenstransfer gilt dabei allzu oft als Einbahnstraße, die von etablierten Demokratien in nichtdemokratische Staaten führt. Durch diese Haltung, die ein Machtgefälle impliziert, wird die Gelegenheit versäumt, von nicht-westlichen Regionen zu lernen, beispielsweise bei der Konfliktüberwindung oder bei innovativen Kommunikationsmodellen. Um unsere eigene Arbeit nicht vorab schon durch eurozentrische Engstirnigkeit zu beschneiden, ist es dringend notwendig, vorhandenes Wissen und Perspektiven aus

\footnotetext{
2 Diese Projekte umfassen bspw. das Projekt „Worlds of Journalism“ an der LMU München, die Graduiertenkollegs School of International and Intercultural Communication (SIIC) und Media Development in the 21st Century (MEDAS-21) an der TU Dortmund, im Verbund mit der RU Bochum und der Universität Duisburg-Essen, das Netzwerk AREACORE (Arab-European Association for Media and Communication Researchers) an der FU Berlin oder das „Media and Transformation“-Projekt an der Universität Erfurt, um nur einige zu nennen.
} 
nicht-westlichen Forschungen einzubeziehen und die eigene Forschung in diesem Kontext zu reflektieren. Die Außensicht kann als eye-opener wesentlich die Innensicht stärken. Der beiderseitige Wissenstransfer und eine inklusive wissenschaftliche Gemeinschaft sind heute unerlässlich, da viele Staaten einerseits mit denselben globalen Herausforderungen zu kämpfen haben, wie beispielsweise der Polarisierung von Gesellschaften, der Erderwärmung oder dem Umgang mit einer Pandemie. Andererseits treten Phänomene zeitlich versetzt und in unterschiedlicher Intensität auf, und es lassen sich nur durch permanente Beobachtung dieser Entwicklungen aussagekräftige Schlussfolgerungen ziehen. Während beispielsweise der traditionelle Journalismus in den USA dramatisch schrumpft, expandiert die Branche in anderen Weltregionen.

Der Akzeptanz internationaler wissenschaftskultureller Diversität mit Blick auf Theorien, Methodologien, aber auch der Zuschreibung von Relevanz an Untersuchungsgegenstände ist nicht länger auszuweichen. Im Gegenteil, es ist die Zeit für einen globalen Dialog gekommen zwischen den zahlreichen, oft stark getrennten lokalen Wissenschaftsgemeinschaften dieser Erde über Universalität und Partikularität unserer wissenschaftlichen Arbeit. Wenn wir diese Aufgabe des globalen Dialogs auf Augenhöhe ernst nehmen und uns nicht vorrangig davon leiten lassen, was für „uns“ (beispielsweise aus sicherheitspolitischen Gründen) relevant zu sein scheint, können wir den Beitrag unserer Wissenschaft zu einer stabilen und nachhaltigen globalen Entwicklung erhöhen. Eine solche Perspektive ist bei Beratungsdienstleistungen für Öffentlichkeit, Medienpraxis, Medien- und Außenpolitik und Diplomatie dringend notwendig.

Für eine kosmopolitische Kommunikationswissenschaft bedeutet das: „Ent-Westernisierung“ und Dezentrierung. Das Denken in vermeintlichen Gegensatzpaaren wie etwa ,the West and the Rest“, Zentrum versus Peripherie, Globaler Norden und Globaler Süden wird mithin obsolet und sollte - wo immer möglich - durch ein plurales oder inklusives Verständnis ersetzt werden. „Wir“ und „die anderen“ waren und sind Konstruktionen.

\section{Wider kommunikationswissenschaftliche terrae incognitae und blind spots}

Die deutsche Kommunikationswissenschaft im internationalen Kontext muss sich neuen Theorieansätzen und Forschungsdesigns auch aus bislang weitgehend unbeachteten Weltregionen öffnen. Internationale Modellierungsversuche von Mediensystemen und Kommunikationskulturen aus westlichen Federn weisen immer noch erhebliche Defizite auf und lassen sich nur äußerst eingeschränkt auf die kommunikationswissenschaftlichen terrae incognitae des Nicht-Westens übertragen.

Westliche normative Ansätze scheitern nicht selten in nicht-westlichen Kontexten - und ironischerweise zuweilen auch im Westen selbst. In Fragen der (auch technologischen) Digitalisierungsprozesse von Medien und Kommunikation sind manche nicht-westliche Länder wiederum einigen Staaten des Globalen Nordens längst Meilen voraus. Um diese Ambivalenzen adäquat verstehen und einordnen zu können, ist es wichtig, der De-Kontextualisierung unserer Wissenschaft bewusst zu 
widerstehen, indem zum Beispiel mehr Formate und Platz in Fachzeitschriften für Kontexterklärungen geschaffen werden. Denn zu oft finden wir in wissenschaftlichen Artikeln zwar methodisch elaborierte Auswertungen von empirischen Daten, aber kein richtiges Wissen darüber, was genau diese empirischen Daten in bestimmten Zusammenhängen bedeuten. Um dieses Kontextwissen zu verbessern, muss die deutsche Kommunikationswissenschaft ihre weltweite Expertise - gerade mit Blick auf viele Länder Asiens, Afrikas und Lateinamerikas und Aspekte wie außereuropäische und außernordamerikanische Strukturbedingungen und Theoriemodelle dringend erweitern und auch strukturell verankern. Damit einher geht auch die Anerkennung einer dynamischen Pluralisierung innerhalb westlicher Nationen. Bisher werden beispielsweise binäre Vorstellungen von „einheimisch“ und „migrantisch“ auch dort fortgeschrieben, wo längst ein ,dazwischen“ der postmigrantischen Gesellschaft stattfindet.

Gerade in solchen kommunikationswissenschaftlichen blind spots werden theoretische und empirisch-methodologische Ansätze entwickelt, die im deutschen Lehrbuchkanon noch nicht angekommen sind. Das liegt auch daran, dass die Forschung zu Medienlandschaften jenseits der westlichen Welt in der deutschen Kommunikationswissenschaft auf institutioneller Ebene trotz der anfangs beschriebenen Öffnungstendenzen ausgesprochen schwach verankert ist. So gibt es, um nur das augenscheinlichste Beispiel zu nennen, derzeit in Deutschland keinen kommunikationswissenschaftlichen Lehrstuhl oder keine Forschergruppe, die sich mit der Medienlandschaft des bevölkerungsstärksten Landes der Erde, China, beschäftigt. Im Gegensatz hierzu sind etwa in Nachbardisziplinen wie der Soziologie oder Politikwissenschaft und dies teils bereits seit Jahrzehnten - eine Reihe von Professuren institutionalisiert, die verschiedene Staaten oder geographische Regionen als explizite Arbeitsschwerpunkte in ihrer Denomination tragen. Diese strukturelle, zweifelsohne ,tiefe Internationalisierung“ des Forschungsgegenstandes ist in der Kommunikationswissenschaft bislang nicht vollzogen. Als Folge mangelt es in Deutschland derzeit nicht nur an kommunikationswissenschaftlicher Expertise zu Medien in nicht-westlichen und transkulturellen Kontexten, sondern ebenso - und dies wiegt langfristig sicherlich schwerer - an Hochschulstrukturen, in denen wissenschaftlicher Nachwuchs diese Expertise erwerben könnte.

\section{Komparative und kollaborative interdisziplinäre Forschung - mehr als Zukunftsmusik}

Eine kosmopolitische Kommunikationswissenschaft benötigt verstärkt komparative und kollaborative interdisziplinäre Forschung. Noch ist sie überwiegend eher rare Ausnahme, mancherorts ist sie aber schon mehr als Zukunftsmusik - auch aus Gründen der Bündelung von Kompetenzen. Komparative und kollaborative Medien- und Kommunikationsforschung schafft globales Wissen und stellt es weltweit zur Verfügung. In einer (digital) vernetzten Weltgesellschaft ist eine Scientific community unerlässlich, die sich einerseits innerhalb der eigenen Disziplin weltweit zugehörig fühlt und gemeinsame Ziele verfolgt, die sich aber andererseits auch nicht hinter 
den Schranken des eigenen Fachs verbarrikadiert, sondern inter- und transdisziplinär operiert.

Die inhaltliche Internationalisierung und transkulturelle Ausrichtung der Kommunikationswissenschaft hat die Erweiterung von Raumgrenzen der untersuchten Gegenstände und deren Kontexte im Blick. Die neue Selbstverständlichkeit von Vergleichen jenseits der herkömmlichen Forschungsrouten hat allerdings nicht automatisch kosmopolitische Nebeneffekte. Ein sinnstiftender Vergleich braucht auch einen konzeptuellen Nährboden interdisziplinärer Aufgeschlossenheit, will er sich nicht in einfachen Ähnlichkeits- und Differenzanalysen erschöpfen. Ohne kompetente Gesellschaftsbeschreibung wird die Interpretation vergleichender Ergebnisse kaum gut, wenn nicht gar unmöglich. Die Wahl der Vergleichskontexte kann noch so anti-stereotyp sein - sie ist sinnlos, wenn die Ergebnisse am Ende in stereotypen Containern kultureller Differenzwahrnehmung landen. Komparatistik lebt von Kontextualisierung.

Daraus folgt, dass die internationale und transkulturelle Kommunikationsforschung gut beraten ist, sich auch mit den Instrumenten der Politikwissenschaft, Soziologie oder der Kulturwissenschaften auszustatten und sich die Zeit zu nehmen, in die dichten Beschreibungen der Area Studies einzusteigen. Anders gewendet heißt das aber auch, dass die Interdisziplinarität der Kommunikationswissenschaft zugleich ihre Internationalität und Transkulturalität vorbereitet. Wenn die Kommunikationswissenschaft neben ihren Kernbereichen zum Beispiel der Massenkommunikation und des Journalismus auch die nicht mediatisierten kommunikativen Prozesse in Politik und Gesellschaft im Blick behält, kann sie der international vergleichenden Kommunikationsforschung den Boden bereiten. Beispiele sind das Verhältnis von öffentlicher und nicht-öffentlicher Kommunikation; das Verständnis der kommunikativen Filterungsprozesse gesellschaftlicher Deutungen; die Identifikation von Meinungsführer*innen oder die kommunikativen Mechanismen sozialer Bewegungen; die Kommunikationsprozesse der Straße und des Marktes, der alten und neuen Salons; die Alltagskommunikation der (post)modernen und postmigrantischen Gruppen und Gemeinschaften in ihren sozialen Rückzugsräumen. Diese kommunikativen Phänomene und sozialen Formationen verstehen wir häufig selbst in uns bekannten Kontexten zu wenig.

Statt also vorschnelle Generalerzählungen mit Universalitätsanspruch von Modernisierung, Globalisierung bis hin zur Digitalisierung zu bemühen, die uns aus westlicher Wissenschaftstradition allzu sehr vertraut sind, sollten vielleicht die feinen Unterschiede kommunikativer Entwicklungen hier wie dort erst einmal dezidiert herausgearbeitet werden, was ohne Kenntnis der historischen Entwicklungen und politischen, sozialen und kulturellen Verhältnisse kaum gelingen kann. Die gleichzeitige Stärkung von Interdisziplinarität und Internationalisierung ergibt also eine „Win-Win“-Situation.

Diese Überlegungen zeigen, dass Internationalisierung kein einfaches „Add-on“ kommunikationswissenschaftlicher Forschung und Lehre sein kann. Komparative Arbeit erfordert Lernprozesse, Zeit und entsprechende Ressourcen. Kooperatives Denken geht nur in Teamarbeit, interdisziplinär wie interregional. Es erfordert zudem Mut, weil internationale Vergleiche und transkulturelle Perspektiven häufig eher 
mit der Suche nach dem bisher Unbekannten als mit dem Testen des Bekannten verbunden sind.

Eine „tiefe Internationalisierung“ sollte also von einem Vertrauen in kontinuierliches Lernen getragen werden, das wiederum nur in stabilen Strukturen entstehen kann. Internationale und transkulturelle Forschung steht aufgrund der geschilderten Anforderungen sicher nicht am Anfang akademischer Biografien, aber sie braucht deswegen umso mehr frühe und kontinuierliche Impulse, die die deutsche Kommunikationswissenschaft konsequenter ernst nehmen sollte.

\section{Fazit und Ausblick: Maßnahmen zur ,tiefen Internationalisierung“}

Wir appellieren an alle in Deutschland tätigen Kommunikationswissenschaftler*innen, also an unsere Scientific community, und besonders an unsere Fachgesellschaft DGPuK, ihre Verantwortung im Bereich der Internationalisierung zu überdenken und den Prozess der ,tiefen Internationalisierung“ in Forschung, Lehre und Hochschulstrukturen voranzutreiben. Diese Neuorientierung ist nicht nur wissenschaftlich relevant, um die Unterrepräsentation weiter Teile der Welt und innerhalb westlicher Gesellschaften als Desiderata zu beschreiben und bestehende Forschungslücken zu schließen. Sie ist auch gesellschaftlich relevant und wird für die Zukunft von allergrößter Bedeutung sein. Die Erforschung der Welt muss heraus aus der Nische des akademischen Spezialistentums und ins Zentrum wissenschaftlichen Arbeitens gerückt werden. Die Globalisierung der Wissenschaft ist kein abgeschlossener Prozess, sondern eine Jahrhundertaufgabe, zu deren Bewältigung wir alle zur Mitwirkung aufrufen. Die Erforschung von nicht-westlichen Ländern sollte nicht nur in Krisenzeiten als relevant wahrgenommen werden.

Insgesamt ist daher nicht nur eine verbesserte strukturelle Absicherung der internationalen Kommunikationswissenschaft an deutschen Hochschulen erforderlich, sondern auch eine Sensibilisierung des Fachs und der deutschen Wissenschaftsgemeinschaft, von der Fachgesellschaft DGPuK bis zu den Herausgeber*innen von in Deutschland erscheinenden Fachzeitschriften und den Verleger*innen von Fachverlagen.

Das Fach kann und muss in puncto Internationalisierung besser werden. Und dies nicht nur in quantitativer Hinsicht (durch Konferenzteilnahmen und mehr Publikationen), sondern auch durch die Wertschätzung der globalen Vielfalt inhaltlicher Problemstellungen und intellektueller Denkrichtungen - über die USA und andere englischsprachige Länder hinaus. Die internationale Kommunikationsforschung sollte mehr als nur ein Nebenschauplatz der Kommunikationswissenschaft sein, denn nur so vermag sie, zentrale Annahmen über Medien und Kommunikationsprozesse, die wir seit langem für selbstverständlich gehalten haben, in Frage zu stellen.

Als Maßnahmen zur ,tiefen Internationalisierung“ der Kommunikationswissenschaft in Deutschland schlagen wir im Einzelnen vor:

1. eine systematische Bestandsaufnahme des aktuellen Stands der internationalen Kommunikationsforschung und -lehre in Deutschland auch im Vergleich zu anderen Ländern; 
2. mehr Investitionen in international, transkulturell und komparativ orientierte Lehrstühle/Professuren und Mitarbeiter*innenstellen an deutschen Hochschulen;

3. verstärkte Appelle von Seiten der Fachgesellschaft DGPuK an hochschulpolitische Verantwortliche in Deutschland und an Hochschulen sowie verstärkte Impulse für internationale Ausschreibungen;

4. die Integration nicht-eurozentrischer Perspektiven in Curricula sowie mehr komparative Lehre;

5. die Gründung eines „Koordinierungszentrums für Internationale Kommunikationswissenschaft".

Funding Open Access funding provided by Projekt DEAL.

Open Access Dieser Artikel wird unter der Creative Commons Namensnennung 4.0 International Lizenz veröffentlicht, welche die Nutzung, Vervielfältigung, Bearbeitung, Verbreitung und Wiedergabe in jeglichem Medium und Format erlaubt, sofern Sie den/die ursprünglichen Autor(en) und die Quelle ordnungsgemäß nennen, einen Link zur Creative Commons Lizenz beifügen und angeben, ob Änderungen vorgenommen wurden.

Die in diesem Artikel enthaltenen Bilder und sonstiges Drittmaterial unterliegen ebenfalls der genannten Creative Commons Lizenz, sofern sich aus der Abbildungslegende nichts anderes ergibt. Sofern das betreffende Material nicht unter der genannten Creative Commons Lizenz steht und die betreffende Handlung nicht nach gesetzlichen Vorschriften erlaubt ist, ist für die oben aufgeführten Weiterverwendungen des Materials die Einwilligung des jeweiligen Rechteinhabers einzuholen.

Weitere Details zur Lizenz entnehmen Sie bitte der Lizenzinformation auf http://creativecommons.org/ licenses/by/4.0/deed.de.

Prof. Dr. C. Richter ist Professorin für Internationale Kommunikation an der FU Berlin. 